\title{
Analysis of 3-hydroxyisovaleric acid and 3-hydroxybutyric acid in plasma samples by LC-MS/MS
}

\author{
Tuba REÇBER ${ }^{1 *}\left(\mathbb{D}\right.$, Ece ÖZKAN 1, 2 (D) , Emirhan NEMUTLU 1 (D) , Mehmet Sinan BEKSAC ${ }^{3}(\mathbb{D}$, \\ Sedef KIR ${ }^{1}$ D
}

1 Department of Analytical Chemistry, Faculty of Pharmacy, Hacettepe University, 06100 Sihhiye, Ankara, Turkey

2 Department of Analytical Chemistry, Faculty of Pharmacy, Başkent University, 06100, Ankara, Turkey

3 Department of Obstetrics and Gynecology, Division of Perinatology, Faculty of Medicine, Hacettepe University, 06100 Sihhiye, Ankara, Turkey

* Corresponding Author. E-mail: tuba.recber@hacettepe.edu.tr (T.R.); Tel. +90-312-305 1499.

Received: 04 September 2021 / Revised: 18 November 2021 / Accepted: 03 December 2021

\begin{abstract}
Down syndrome is a common genetic disorder that results from the presence of an extra chromosome in the $21^{\text {st }}$ chromosome pair of humans. Metabolomics is an alternative method in discovery of new biomarkers for the screening and diagnosis of Down syndrome. In this study, quantitative analyzes of 3-hydroxybutyric acid and 3hydroxyisovaleric acid, selected as possible markers for prenatal diagnosis of Down syndrome were performed. LCMS/MS analyzes were performed on a Phenomenex Luna $\mathrm{NH}_{2}(100 \times 4.6 \mathrm{~mm}, 3 \mu \mathrm{m})$ column using a mobile phase mixture of $0.1 \%$ formic acid and acetonitrile containing $0.1 \%$ formic acid at a flow rate of $0.35 \mathrm{~mL} / \mathrm{minute}$. The MRM transitions were $103.0 \rightarrow 59.0$ for 3-hydroxybutyric acid and $117.1 \rightarrow 59.0$ for 3-hydroxyisovaleric acid. Under these conditions, the retention times of 3-hydroxyisovaleric acid 3-hydroxybutyric acid were 2.7 and 3.1 minute, respectively. The method was found linear from 0.1 to $10.0 \mu \mathrm{g} / \mathrm{mL}$ for both metabolites. The limit of detection (LOD) was 0.017 $\mu \mathrm{g} / \mathrm{mL}$ for 3-hydroxybutyric acid and $0.003 \mu \mathrm{g} / \mathrm{mL}$ for 3-hydroxyisovaleric acid. The lower limit quantification (LLOQ) was $0.045 \mu \mathrm{g} / \mathrm{mL}$ for 3-hydroxybutyric acid and $0.008 \mu \mathrm{g} / \mathrm{mL}$ for 3-hydroxyisovaleric acid. The method has been proven to be selective, precise, accurate, sensitive, and robust based on the validation studies results. Finally, the method was applied to plasma samples of the pregnant women with healthy fetus $(n=30)$ and with Down syndrome fetus (n $=17)$. As a result of the analysis, a statistically significant increase $(\mathrm{p}<0.01)$ was observed in the 3-hydroxybutyric acid level of the group with Down syndrome compared to the healthy group. This result strengthens the use of 3hydroxybutyric acid as an important biomarker in the prenatal screening/diagnosis of Down syndrome.
\end{abstract}

KEYWORDS: Down syndrome; prenatal screening; metabolomics; liquid chromatography-tandem mass spectrometry (LC-MS/MS); 3-hydroxyisovaleric acid; 3-hydroxybutyric acid.

\section{INTRODUCTION}

Occurring in about 1 of every 1000-1100 births worldwide, Trisomy 21 (Down syndrome) is the most common chromosomal disorder associated with intellectual disability, craniofacial dysmorphisms and cardiovascular defects [1]. Today, routine screening tests for Down syndrome in pregnancy have become a clinical care standard in developed countries. For the diagnosis of Down syndrome, invasive methods such as Chorionic Villus Biopsy (CVS) and chromosome count in amniocentesis fluid are used [2]. However, noninvasive methods are needed since these invasive methods for the diagnosis of Down syndrome carry the risk of early termination of pregnancy (miscarriage). Advanced metabolomic analyzes is an alternative approach in determining new biomarkers for the screening and diagnosis of Down syndrome.

Metabolomic profiling of Down syndrome was carried out using different analytical methods [3-5]. In these studies, it was stated that metabolites such as 3-hydroxybutyric acid, 2-hydroxybutyric acid, 2ketoisocaproic acid, 3-hydroxyisovaleric acid, threonic acid and oxalic acid were at abnormal levels in pregnant women with Down syndrome.

The biomarker candidates determined with untargeted metabolomic studies have a high potential for the prenatal screening of Down syndrome. However, before clinical application of these metabolites as biomarkers, the results must be verified with cross validation studies by using targeted metabolomic analyzes.

How to cite this article: Reçber T, Özkan E, Nemutlu E, Beksaç MS, Kır S. Analysis of 3-hydroxyisovaleric acid and 3-hydroxybutyric acid in plasma samples by LC-MS/MS. J Res Pharm. 2022; 26(1): 136-144. 
In this study, a liquid chromatography-tandem mass spectrometry (LC-MS/MS) method for two biomarker candidates (3-hydroxybutyric acid and 3-hydroxyisovaleric acid) from the literature for the screening/diagnosis of Down syndrome was developed, validated and applied to plasma samples.

In the literature, there are analyzes of these metabolites with NMR, GC-MS and HPLC methods [6-11]. However, these methods have some disadvantages such as the need for complex, costly and time-consuming sample preparation procedures, low sensitivity and requiring column-switching system for some methods. These limitations can be minimized by using LC-MS/MS methods for quantification. Several LC-MS/MS methods have been reported for the single determination of 3-hydroxybutyric acid [12-14] and 3hydroxyisovaleric acid [15, 16] in biological samples. A study on the simultaneous analysis of 3hydroxybutyric acid and 3-hydroxyisovaleric acid with the GC-MS method has been reported [11]. In this GCMS method, a time-consuming derivatization process and a relatively long analysis time are required for the analysis of metabolites. However, there is no a validated LC-MS/MS method for the simultaneous quantitative analysis of 3-hydroxybutyric acid and 3-hydroxyisovaleric acid from plasma samples in the literature. It has been observed that the proposed LC-MS/MS method has advantages such as ease of sample preparation, analysis time and sensitivity compared to the other methods.

\section{RESULTS AND DISCUSSION}

\subsection{Mass spectrometry}

The chromatographic conditions and the mass spectrometric parameters were adjusted for optimum detection of 3-hydroxybutyric acid and 3-hydroxyisovaleric acid. Firstly, optimization of MRM parameters was performed by injecting 3-hydroxybutyric acid $(1 \mu \mathrm{g} / \mathrm{mL})$ and 3-hydroxyisovaleric acid solutions (1 $\mu \mathrm{g} / \mathrm{mL}$ ) directly into the MS system. The precursor ions were selected for each substance (103.0 for 3hydroxybutyric acid and 117.1 for 3-hydroxyisovaleric acid). After collision-induced dissociation, the most abundant product ions were at m/z 103.0 for 3-hydroxybutyric acid and at m/z 117.1 for 3-hydroxyisovaleric acid using $11 \mathrm{mV}$ collision energy (Figure 1). For the quantitative analysis, the MRM transitions were selected $113.0 \rightarrow 59.0$ for 3-hydroxybutyric acid and $117.1 \rightarrow 59.0$ for 3-hydroxyisovaleric acid.
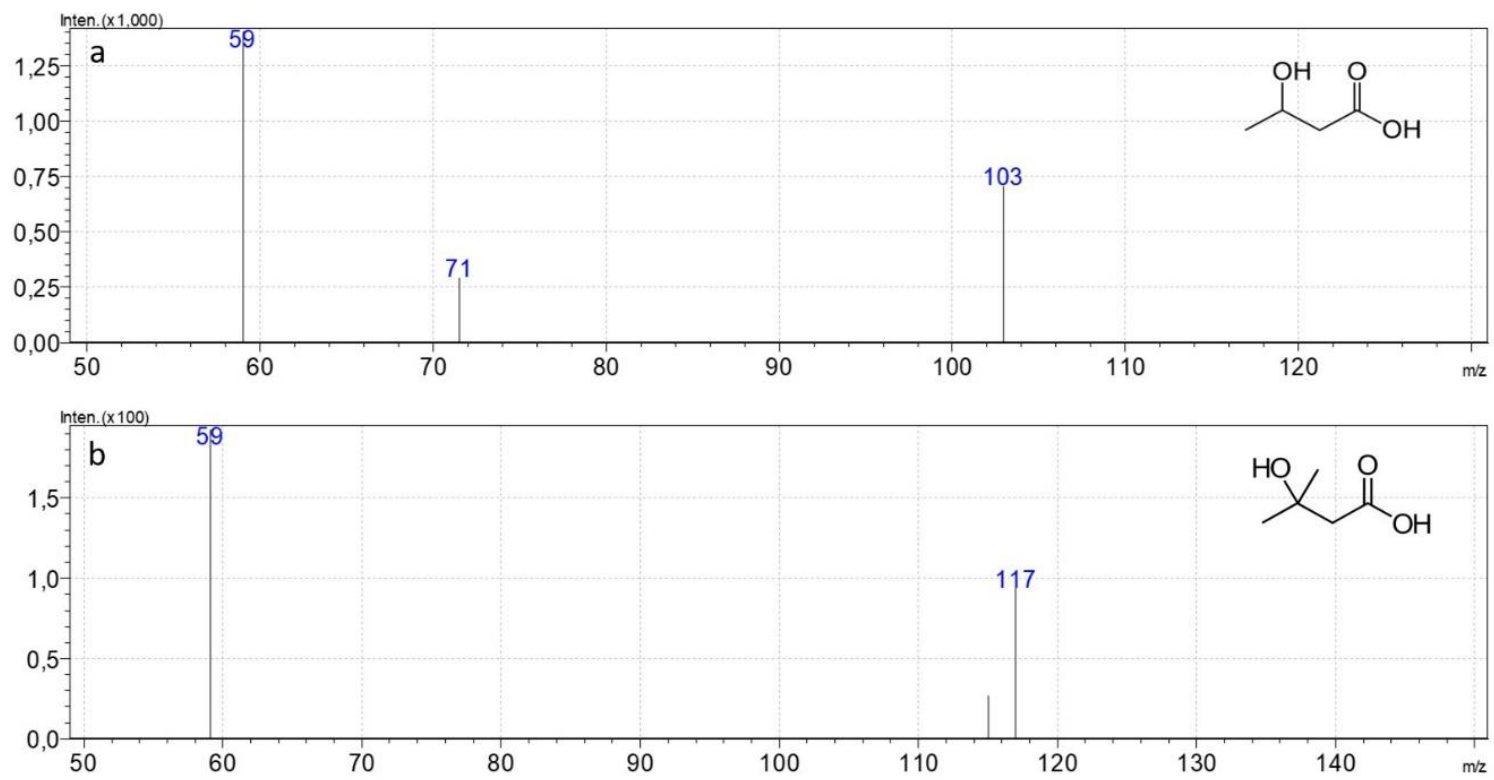

Figure 1. MS/MS fragmentation pattern; a) 3-hydroxybutyric acid and b) 3-hydroxyisovaleric acid.

To achieve the optimum chromatographic conditions, various columns (C18, $\mathrm{HILIC}$, and $\mathrm{NH}_{2}$ ), organic solvents (acetonitrile and methano)l and different solutions (1 $\mathrm{mM}$ ammonium format, $1 \mathrm{mM}$ ammonium acetate, and $0.1 \%$ formic acid) were tested. First, the C18 column was tested for separation and it was observed that the metabolites were not retained on the column and eluted at dead volume. Then the column was switched with a HILIC column and analysis were performed. This time metabolites were retained, but the peak symmetries of the metabolites were not acceptable for system suitability limits. Then another polar column an $\mathrm{NH}_{2}$ column $(100 \times 4.6 \mathrm{~mm}, 3 \mu \mathrm{m})$ was tested and chosen as stationary phase due to symmetric peaks for 3-hydroxybutyric acid and 3-hydroxyisovaleric acid and short analysis time. 
Various combinations of the buffers and the organic solvents were investigated and compared for both metabolites in terms of sensitivity, efficiency and peak shape. $0.1 \%$ formic acid and acetonitrile containing $0.1 \%$ formic acid was selected as optimal mobile phase. For elution of metabolites, a gradient elution with water containing $0.1 \%$ formic acid as solvent $\mathrm{A}$ and acetonitrile containing $0.1 \%$ formic as solvent $\mathrm{B}$ was applied. The total run time was $10 \mathrm{~min}$ and the retention time of 3-hydroxyisovaleric acid and 3hydroxybutyric acid were $2.7 \mathrm{~min}$ and $3.1 \mathrm{~min}$, respectively (Figure 2).

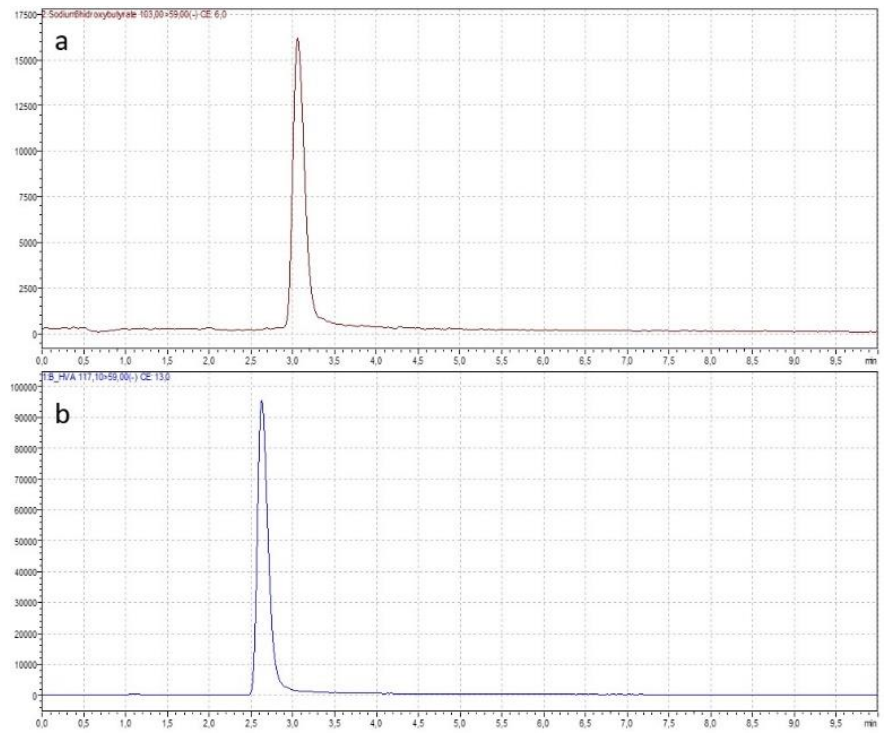

Figure 2. Representative chromatograms; a) 3-hydroxybutyric acid (1.0 $\mu \mathrm{g} / \mathrm{mL})$ and b) 3-hydroxyisovaleric acid $(1.0 \mu \mathrm{g} / \mathrm{mL})$ at optimum chromatographic conditions.

\subsection{System suitability}

The system suitability of the developed method under optimum analysis conditions was determined in terms of column efficiency (theoretical plate number, $\mathrm{N})$, capacity factor $\left(\mathrm{k}^{\prime}\right)$, tailing factor, resolution (Rs), and selectivity factor (a) parameters. The results of system suitability test are given in Table 1.

Table 1. System suitability parameters for 3-hydroxybutyric acid and 3-hydroxyisovaleric acid $(n=6)$.

\begin{tabular}{llll}
\hline & $\begin{array}{l}\text { 3-hydroxybutyric } \\
\text { acid }\end{array}$ & $\begin{array}{l}\text { 3-hydroxyisovaleric } \\
\text { acid }\end{array}$ & $\begin{array}{l}\text { Requirements } \\
\text { (17) }\end{array}$ \\
\hline Injection repeatability (RSD of $\mathrm{t}_{\mathrm{R}}$ ) & $0.09 \%$ & $0.12 \%$ & $<1 \%$ \\
Theoretical plate number $(\mathrm{N})$ & 22775 & 17963 & $>2000$ \\
Capacity factor $\left(\mathrm{k}^{\prime}\right)$ & 2.80 & 2.27 & $1<\mathrm{k}^{\prime}<5$ \\
Tailing factor & 1.324 & 1.385 & $\mathrm{~T}<2$ \\
Resolution $(\mathrm{Rs})$ & & 3.95 & $>1.5$ \\
Selectivity factor $(\mathrm{a})$ & & 1.23 & $>1$ \\
\hline
\end{tabular}

\subsection{Method validation}

The optimized method was validated in terms of linearity, sensitivity, matrix effect, carry over, precision, accuracy, selectivity, and ruggedness according to ICH validation guidelines for bioanalytical methods [17].

\subsubsection{Linearity}

Calibration solutions at concentrations of $0.1,0.3,0.5,0.8,1,2,5$, and $10 \mu \mathrm{g} / \mathrm{mL}$ were prepared. The calibration curves were created by plotting the peak area of 3-hydroxybutyric acid and 3-hydroxyisovaleric acid versus their concentrations. The regression equations were determined from the calibration graphs. The method showed an acceptable linearity range from 0.1 to $10 \mu \mathrm{g} / \mathrm{mL}$ for 3-hydroxybutyric acid and 3hydroxyisovaleric acid (Table 2). 
Table 2. Linearity and sensitivity of the method for 3-hydroxybutyric acid and 3-hydroxyisovaleric acid $(\mathrm{n}=7)$.

\begin{tabular}{lcc}
\hline & 3-hydroxybutyric acid & 3-hydroxyisovaleric acid \\
\hline Regression equation $^{\mathrm{a}}$ & $\mathrm{y}=36736 \mathrm{x}+4466.5$ & $\mathrm{y}=176947 \mathrm{x}+47858$ \\
Determination coefficient $\left(\mathrm{R}^{2}\right)$ & 0.998 & 0.998 \\
Linearity range $(\mu \mathrm{g} / \mathrm{mL})$ & $0.1-10.00$ & $0.1-10.00$ \\
Limit of detection $(\mathrm{LOD})(\mu \mathrm{g} / \mathrm{mL})$ & 0.017 & 0.003 \\
Lower limit of quantification $(\mathrm{LLOQ})(\mu \mathrm{g} / \mathrm{mL})$ & 0.045 & 0.008 \\
\hline
\end{tabular}

\subsubsection{Sensitivity}

LOD and LLOQ of the developed method were determined as concentration values at which the signalto-noise ratio is 3:1 and 10:1, respectively. According to the results (Table 2) the method was sensitive for estimating 3-hydroxybutyric acid and 3-hydroxyisovaleric acid in human plasma.

\subsubsection{Matrix effect}

To examine the effect of the sample matrix on the ionization efficiency of 3-hydroxybutyric acid and 3hydroxyisovaleric acid, standard solutions were added to both water and pooled plasma sample at two different concentrations. The peak areas of plasma and water spiked were compared and matrix effect was calculated as 100.53\% (CV\%: 4.74) for 3-hydroxybutyric acid and 90.69\% (CV\%: 0.63) for 3-hydroxyisovaleric acid. The results show that the sample matrix has no effect on the ionization and fragmentation of metabolites.

\subsubsection{Dilution integrity}

To test dilution integrity, the stock solutions of 3-hydroxybutyric acid and 3-hydroxyisovaleric acid were spiked into pooled plasma at a concentration equivalent to three times of the upper limit of quantification $(30 \mu \mathrm{g} / \mathrm{mL})$ and then diluted 1:5 and 1:10 with water. Each of the diluted samples (n: 6) was analyzed by the LC-MS/MS method and compared to the nominal concentrations. Dilution integrity was found with in accuracy and precision values of $\leq 9.16 \%$ and $\leq 10.82 \%$ for the 5 -fold and 10 -fold dilution, respectively.

\subsubsection{Carry-over}

Carry-over effect was determined by injection of the mobile phase following by injections of the highest concentration solutions $(10 \mu \mathrm{g} / \mathrm{mL})$ of both metabolites. The carryover of the developed method was lower than $20 \%$ of LLOQ values.

\subsubsection{Precision}

The precision of the developed method was evaluated by injection repeatability and intra- and interday precision studies.

For injection repeatability, 3-hydroxybutyric acid and 3-hydroxyisovaleric acid solutions prepared at a concentration of $1.00 \mu \mathrm{g} / \mathrm{mL}$ were analyzed by six consecutive injections. The RSD of the metabolites peak areas were found $0.02 \%$ for 3-hydroxybutyric acid and $0.03 \%$ for 3-hydroxyisovaleric acid.

The intra-day and inter-day analyzes were evaluated by six replicate analyzes at four concentration levels (LLOQ, 0.5, 1.0, and $5.0 \mu \mathrm{g} / \mathrm{mL}$ ) in the linearity range of 3-hydroxybutyric acid and 3-hydroxyisovaleric acid on the same day and on six different days, respectively (Table 3 ).

\subsubsection{Accuracy}

The intra-day and inter-day accuracy analyzes were evaluated by six replicate analyzes at four concentration levels (LLOQ, $0.5,1.0$, and $5.0 \mu \mathrm{g} / \mathrm{mL}$ ) in the linearity range of 3-hydroxybutyric acid and 3hydroxyisovaleric acid on the same day and on six different days, respectively (Table 3).

The accuracy of the developed method was also evaluated with recovery studies. Standard solutions of both metabolites were spiked into water and pooled plasma samples, the samples were analyzed under optimum experimental conditions. The calibration curves for water and plasma spiked were plotted and 
regression equation were calculated. The recovery was determined as $93.22 \%$ for 3-hydroxybutyric acid and $95.26 \%$ for 3-hydroxyisovaleric acid by comparing the slopes of the calibration curves.

Table 3. Intra- and inter-day accuracy and precision of the developed method.

\begin{tabular}{lcccc}
\hline & $\begin{array}{c}\text { Added } \\
(\mu \mathrm{g} / \mathrm{mL})\end{array}$ & $\begin{array}{c}\text { Found } \\
(\mu \mathrm{g} / \mathrm{mL})\end{array}$ & $\begin{array}{c}\text { Precision } \\
\mathbf{C V} \%\end{array}$ & $\begin{array}{c}\text { Accuracy } \\
\text { Bias } \%\end{array}$ \\
\hline Intra-day $(\mathbf{n}=\mathbf{6})$ & & & & \\
3-hydroxybutyric acid & 0.045 & $0.045 \pm 0.01$ & 2.23 & 1.11 \\
& 0.50 & $0.50 \pm 0.01$ & 1.43 & 0.53 \\
& 1.00 & $0.99 \pm 0.01$ & 2.11 & -1.12 \\
3-hydroxyisovaleric acid & 5.00 & $4.95 \pm 0.02$ & 1.03 & 1.04 \\
& 0.008 & $0.007 \pm 0.01$ & 2.01 & -2.13 \\
& 0.50 & $0.47 \pm 0.01$ & 2.88 & -5.75 \\
Inter-day (n = 6) & 1.00 & $1.01 \pm 0.02$ & 4.43 & 0.76 \\
3-hydroxybutyric acid & 5.00 & $4.84 \pm 0.02$ & 0.98 & -3.17 \\
& & & & \\
& 0.045 & $0.046 \pm 0.03$ & 4.25 & 2.73 \\
& 0.50 & $0.49 \pm 0.01$ & 2.89 & $-2,17$ \\
3-hydroxyisovaleric acid & 1.00 & $0.99 \pm 0.01$ & 1.45 & -1.01 \\
& 5.00 & $4.96 \pm 0.06$ & 3.08 & -0.84 \\
& 0.008 & $0.008 \pm 0.04$ & 3.11 & 2.05 \\
& 0.50 & $0.48 \pm 0.01$ & 3.67 & -4.69 \\
& 1.00 & $1.02 \pm 0.02$ & 4.66 & 1.96 \\
& 5.00 & $4.98 \pm 0.05$ & 2.23 & -0.45 \\
\hline
\end{tabular}

a Mean \pm standard error $(n=6)$.

\subsubsection{Selectivity}

In the selectivity study, chromatograms of standard solutions and plasma solutions for both metabolites were examined. In optimum analysis conditions, it was determined that only one peak occurred in retention times of metabolite peaks, there was no change in retention times with increasing metabolite concentration, and metabolite peaks were observed only at specific $\mathrm{m} / \mathrm{z}$ values. Moreover, the slope of the calibration curves of plasma and water spiked standard were similar to each other. Therefore, it can be said that the LC-MS/MS method is selective for 3-hydroxybutyric acid and 3-hydroxyisovaleric acid.

\subsubsection{Ruggedness}

The ruggedness of the method was evaluated with two analysts at a concentration level $(1.00 \mu \mathrm{g} / \mathrm{mL})$. The results of the analysts were compared and the mean of the results were not statistically different (Table $4)$.

Table 4. Ruggedness data of the developed method $(n=6)$.

\begin{tabular}{ccc}
\hline & 3-hydroxybutyric acid & 3-hydroxyisovaleric acid \\
\cline { 2 - 3 } & Concentration $(\mu \mathrm{g} / \mathrm{mL})$ & Concentration $(\mu \mathrm{g} / \mathrm{mL})$ \\
Analyst 1 & $0.96 \pm 0.01$ & $1.09 \pm 0.02$ \\
Analyst 2 & $0.99 \pm 0.03$ & $1.14 \pm 0.01$ \\
p values & 0.13 & 0.09 \\
\hline
\end{tabular}

\subsubsection{Stability}

The stability of 3-hydroxybutyric acid and 3-hydroxyisovaleric acid was investigated using plasma samples (spiked with $1.0 \mu \mathrm{g} / \mathrm{mL}$ standard solution) for different conditions such as short term (room temperature for $24 \mathrm{~h}$ ), autosampler (room temperature for $8 \mathrm{~h}$ ), long term (-20॰C for 10 days) and freeze-thaw cycles ( 3 cycles). The results were compared with the analysis results of freshly prepared plasma solutions. Under these conditions, recovery values of 3-hydroxybutyric acid and 3-hydroxyisovaleric acid were found to 
98.1\% and $97.4 \%$ for short term, $92.5 \%$ and $93.8 \%$ for autosampler, $99.2 \%$ and $98.1 \%$ for long term and $97.2 \%$ and $98.0 \%$ at third freeze-thaw cycles, respectively.

\subsection{Assay application in quantification of clinical human serum samples}

The validated LC-MS/MS method was used for the quantitation of 3-hydroxybutyric acid and 3hydroxyisovaleric acid in the plasma samples of 17 pregnant women with Down syndrome and 30 healthy pregnant women (Figure 3-4). The average amount of 3-hydroxybutyric acid and 3-hydroxyisovaleric acid for healthy and diagnosed with Down syndrome pregnant women were shown in Figure 5. Plasma levels of both metabolites increased in the group with Down syndrome compared to the healthy group, as in studies performed with different analysis methods in the literature [11, 18].

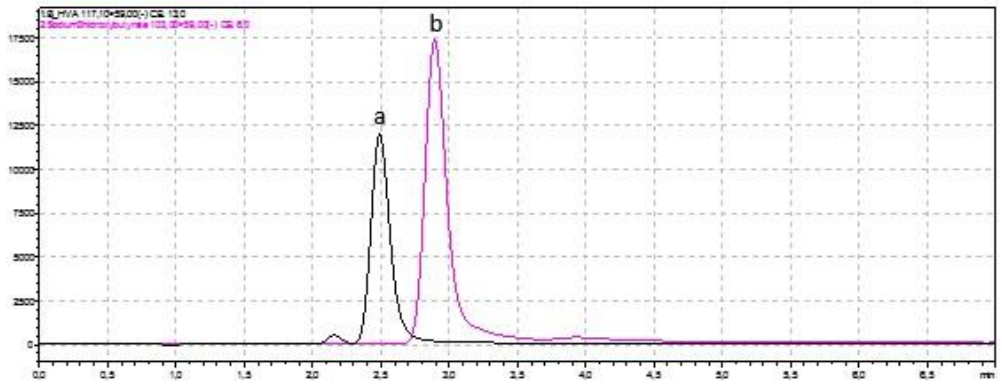

Figure 3. Representative chromatograms of plasma analysis of pregnant women with Down syndrome (a: 3-hydroxyisovaleric acid and b: 3-hydroxybutyric acid)

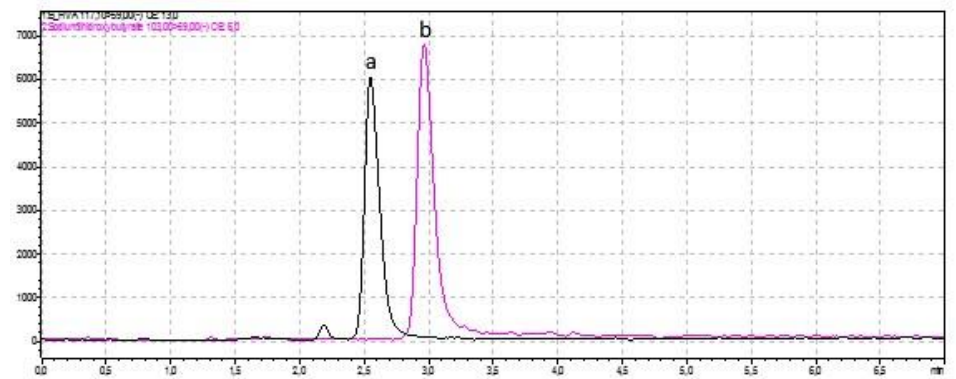

Figure 4. Representative chromatograms of plasma analysis of healthy pregnant women (a: 3hydroxyisovaleric acid and b: 3-hydroxybutyric acid).
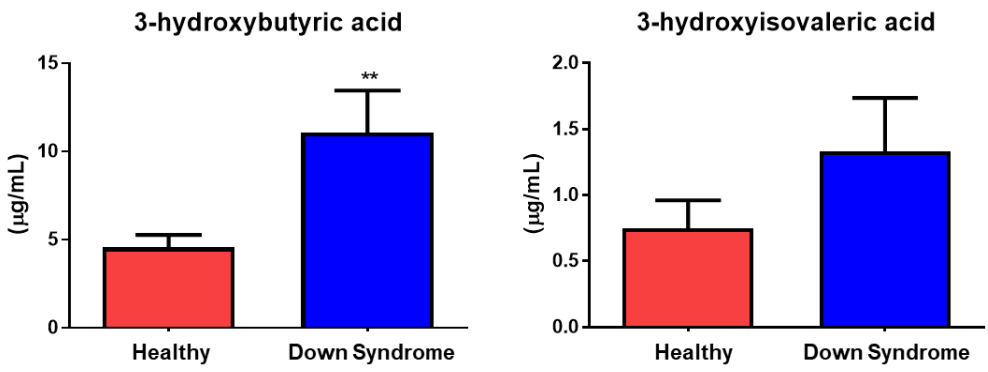

Figure 5. Metabolite levels in plasma samples of pregnant women with Down syndrome and healthy groups $\left({ }^{* *} \mathrm{p}<0,01\right)$.

\section{CONCLUSION}

In this study, an LC-MS/MS method was developed for the quantitation of the two metabolites proposed to be used in the screening/diagnosis of Down syndrome. The developed method was validated by examining the linearity, sensitivity, precision, accuracy, selectivity, and ruggedness parameters. The method was found linear, sensitive, precise, accurate, selective, and rugged according to ICH bioanalytical method guideline. 
The developed and validated method was successfully applied to a total of 47 plasma samples, including 30 healthy and 17 Down syndrome. For 3-hydroxybutyric acid, the difference between the Down syndrome group and the healthy group was statistically significant $(\mathrm{p}<0.01)$. This important difference suggests that 3-hydroxybutyric acid can be an important biomarker and can be used for diagnosis of Down syndrome.

\section{MATERIALS AND METHODS}

\subsection{Chemicals and reagents}

3-hydroxybutyric acid and 3-hydroxyisovaleric acid were purchased from Sigma Aldrich (Saint Louis, MO, USA). Water and acetonitrile were purchased from Merck, while formic acid from Fluka (St. Gallen, Swiss). Solvents used for chromatography were of LC-MS grade.

\subsection{Standard solution preparation}

The standard stock solutions of 3-hydroxybutyric acid and 3-hydroxyisovaleric acid $(1000 \mu \mathrm{g} / \mathrm{mL})$ was prepared in water and methanol, respectively. All stock solutions were stored in the refrigerator. Standard solutions were daily prepared by dilution of the stock solution with mobile phase solution.

\subsection{Patients characteristics}

This study was consisted of 47 pregnancies who underwent invasive prenatal testing (amniocentesis or chorionic villous sampling) for the prenatal diagnosis of chromosomal abnormalities. Blood samples were obtained prior to invasive prenatal testing. As a result of genetic analysis (fetal karyotyping), 17 cases were found to be trisomy-21 (Down syndrome). The karyotyping results of the remaining 30 cases were normal.

\subsection{Ethics statement}

The study was conducted in accordance with the ethical rules of the Helsinki Declaration and Good Clinical Practice. This study was approved by the Ethics Committee of Non-Interventional Clinical Research at the Hacettepe University (GO 14/173). An informed consent form for research purposes was signed by all participants before their participation to the study.

\subsection{Sample preparation}

The blood samples of pregnant women (30 healthy and 17 Down syndrome) were centrifugated at 3000 $\mathrm{rpm}$ for $10 \mathrm{~min}$. Then the plasma samples were stored at $-20^{\circ} \mathrm{C}$ until the analysis. A $100 \mu \mathrm{L}$ of plasma samples were taken and diluted with $200 \mu \mathrm{L}$ of water then an $800 \mu \mathrm{L}$ of acetonitrile was added for protein precipitation. They were vortexed for 1 minute and centrifuged at $15000 \mathrm{rpm}$ for 10 minutes. A $350 \mu \mathrm{L}$ supernatant was taken and diluted with same amount of mobile phase mixture [0.1\% formic acid and acetonitrile containing $0.1 \%$ formic acid (1: 1, v/v)] and injected into the LC-MS/MS.

\subsection{Instrumentation}

The LC-MS/MS system is consisted of a Shimadzu LC-20AXR system coupled to a Shimadzu 8030 MS/MS triple quadrupole tandem mass spectrometer. The mass spectrometric detection was operated in a negative electrospray ionization and multiple reaction monitoring (MRM) mode. Data acquisition and processing were carried out using the Lab Solution Software.

\subsection{LC-MS/MS analysis conditions}

The experiments were performed using a Phenomenex Luna $\mathrm{NH}_{2}$ column $(100 \times 4.6 \mathrm{~mm}, 3 \mu \mathrm{m})$ at a flow rate of $0.35 \mathrm{~mL} \mathrm{~min}^{-1}$ and $40{ }^{\circ} \mathrm{C}$. A gradient elution program with mobile phases $\mathrm{A}(0.1 \%$ formic acid $)$ and $\mathrm{B}$ (acetonitrile containing $0.1 \%$ formic acid) was run. The gradient elution was applied to the mobile phase as follows: from 0 to $1 \mathrm{~min}$ the mobile phase contained $80 \% \mathrm{~B}$; from 1 to $8 \mathrm{~min}$ the percentage of $\mathrm{B}$ decreased to $20 \%$; from 8 to 12 min the B percentage increased to $80 \%$.

The ESI-MS/MS conditions were as follows: negative ion mode; interface voltage, $4.5 \mathrm{kV}$; dwell time, $100 \mathrm{~ms}$; Q1 pre-rod bias voltage were $13 \mathrm{~V}$ for 3-hydroxybutyric acid and $6 \mathrm{~V}$ for 3-hydroxyisovaleric acid; Q3 pre-rod bias voltage were $21 \mathrm{~V}$ for 3-hydroxybutyric acid and $23 \mathrm{~V}$ for 3-hydroxyisovaleric acid; collision energies were $11 \mathrm{eV}$ for both metabolites. The transitions $103.0 \rightarrow 59.0 \mathrm{~m} / \mathrm{z}$ and $117.1 \rightarrow 59.0 \mathrm{~m} / \mathrm{z}$ were used to monitor 3-hydroxybutyric acid and 3-hydroxyisovaleric acid, respectively. Collision activated dissociation 
(CAD) gas (argon) served as medium (arbitrary units) and nebulizer at a gas flow rate of $3 \mathrm{~L} / \mathrm{min}$, and a drying gas flow rate of $15 \mathrm{~L} / \mathrm{min}$, desolvation line temperature of $250{ }^{\circ} \mathrm{C}$, and heat block temperature of $400{ }^{\circ} \mathrm{C}$ were set.

Acknowledgements: This research was supported by Hacettepe University Scientific Research Project Coordination Unit (Project no: 01406301 001-585).

Author contributions: Concept - T.R., E.N., M.S.B., S.K.; Design - T.R., E.N., S.K.; Supervision - T.R., E.N., S.K.; Resource - T.R., E.N., E.Ö., M.S.B., S.K.; Materials - E.N., M.S.B., S.K.; Data Collection \&/or Processing - T.R.; Analysis \&/or Interpretation - T.R.; Literature Search - T.R., E.N.; Writing - T.R., E.N., S.K.; Critical Reviews - T.R., E.Ö., E.N., M.S.B., S.K.

Conflict of interest statement: The authors declared no conflict of interest in the manuscript.

Ethics committee approval: This study was approved by the Ethics Committee of Non-Interventional Clinical Research at the Hacettepe University (GO 14/173).

\section{REFERENCES}

[1] Gardiner K, Herault Y, Lott IT, Antonarakis SE, Reeves RH, Dierssen M. Down syndrome: from understanding the neurobiology to therapy. J Neurosci. 2010; 30(45): 14943-14945. [CrossRef]

[2] Antonarakis SE, Skotko BG, Rafii MS, Strydom A, Pape SE, Bianchi DW, Sherman SL, Reeves RH. Down syndrome. Nat Rev Dis Primers. 2020; 6(1): 1-20. [CrossRef]

[3] Bahado-Singh RO, Akolekar R, Mandal R, Dong E, Xia J, Kruger M, Wishar DS, Nicolaides K. Metabolomic analysis for first-trimester Down syndrome prediction. Am J Obstet Gynecol. 2013; 208(5): 371-378. [CrossRef]

[4] Nemutlu E, Orgul G, Recber T, Aydin E, Ozkan E, Turgal M, Alikasifoglu M, Kır S, Beksac MS. Metabolic infrastructure of pregnant women with trisomy 21 fetuses; metabolomic analysis. Z. Geburtshilfe Neonatol. 2019; 223(05): 297-303. [CrossRef]

[5] Sánchez-Ribas I, Riqueros M, Vime P, Puchades-Carrasco L, Jönsson T, Pineda-Lucena A, Ballesteros A, Domínguez F, Simón C. Differential metabolic profiling of non-pure trisomy 21 human preimplantation embryos. Fertil Steril. 2012; 98(5): 1157-1164. [CrossRef]

[6] Engelke UF, Kremer B, Kluijtmans LA, van der Graaf M, Morava E, Loupatty FJ, Wanders RJA, Moskau D, Loss S, Bergh E, Wevers RA. NMR spectroscopic studies on the late onset form of 3-methylglutaconic aciduria type I and other defects in leucine metabolism. NMR Biomed. 2006; 19(2): 271-278. [CrossRef]

[7] Bahado-Singh RO, Akolekar R, Mandal R, Dong E, Xia J, Kruger M, Wishart D, Nicholadies K. Metabolomics and first-trimester prediction of early-onset preeclampsia. J. Matern.-Fetal Neonatal Med. 2012; 25(10): $1840-1847$. [CrossRef]

[8] Luís PB, Ruiter JP, IJlst L, Diogo L, Garcia P, de Almeida IT, Duran M, Wanders RJ, Silva MFB. Inhibition of 3methylcrotonyl-CoA carboxylase explains the increased excretion of 3-hydroxyisovaleric acid in valproate-treated patients. J Inherit Metab Dis. 2012; 35(3): 443-449. [CrossRef]

[9] Mock DM, Henrich CL, Carnell N, Mock NI. Indicators of marginal biotin deficiency and repletion in humans: validation of 3-hydroxyisovaleric acid excretion and a leucine challenge. Am J Clin Nutr. 2002; 76(5): 1061-1068. [CrossRef]

[10] Cevasco G, Piątek AM, Thea S. HPLC determination of D-3-hydroxybutyric acid by derivatization with a benzofurazan reagent and fluorescent detection: application in the analysis of human plasma. Clin Chim Acta. 2014; 429: 90-95. [CrossRef]

[11] Özkan E, Nemutlu E, Beksac MS, Kır S. GC-MS analysis of seven metabolites for the screening of pregnant women with Down syndrome fetuses. J Pharm Biomed. 2020; 188: 113427. [CrossRef]

[12] Miyazaki T, Honda A, Ikegami T, Iwamoto J, Monma T, Hirayama T, Saito Y, Yamashita K, Matsuzaki Y. Simultaneous quantification of salivary 3-hydroxybutyrate, 3-hydroxyisobutyrate, 3-hydroxy-3-methylbutyrate, and 2-hydroxybutyrate as possible markers of amino acid and fatty acid catabolic pathways by LC-ESI-MS/MS. Springerplus. 2015; 4(1): 1-18. [CrossRef]

[13] Zhang Q, Ford LA, Goodman KD, Freed TA, Hauser DM, Conner JK, Vroom KET, Toal DR. LC-MS/MS method for quantitation of seven biomarkers in human plasma for the assessment of insulin resistance and impaired glucose tolerance. J Chromatogr B. 2016; 1038: 101-108. [CrossRef] 
[14] Reçber T, Nemutlu E, Beksaç K, Aksoy S, Kır S. Optimization and validation of a HILIC-LC-ESI-MS/MS method for the simultaneous analysis of targeted metabolites: Cross validation of untargeted metabolomic studies for early diagnosis of breast cancer. Microchem J. 2020; 159: 105559. [CrossRef]

[15] Horvath TD, Matthews NI, Stratton SL, Mock DM, Boysen G. Measurement of 3-hydroxyisovaleric acid in urine from marginally biotin-deficient humans by UPLC-MS/MS. A Anal. Bioanal Chem. 2011; 401(9): 2805-2810. [CrossRef]

[16] Perry CA, West AA, Gayle A, Lucas LK, Yan J, Jiang X, Malysheva O, Caudill MA. Pregnancy and lactation alter biomarkers of biotin metabolism in women consuming a controlled diet. J Nutr. 2014; 144(12): 1977-1984. [CrossRef]

[17] Bioanalytical Method Validation M10. International Council for Harmonisation of Technical Requirements for Pharmaceuticals for Human Use, draft version 2019.

[18] Bahado-Singh RO, Akolekar R, Chelliah A, Mandal R, Dong E, Kruger M, Wishart DS, Nicolaides K. Metabolomic analysis for first-trimester trisomy 18 detection. Am J Obstet Gynecol. 2013; 209(1): 65-69. [CrossRef]

This is an open access article which is publicly available on our journal's website under Institutional Repository at http://dspace.marmara.edu.tr. 